\title{
All-optical logic devices based on black arsenic-phosphorus with strong nonlinear optical response and high stability
}

\author{
Leiming Wu ${ }^{1,2,4 \dagger}$, Taojian $\mathrm{Fan}^{1 \dagger}{ }^{1 \dagger}$, Songrui Wei ${ }^{1 \dagger}$, Yijun $\mathrm{Xu}^{1}$, Ye Zhang ${ }^{1}$, \\ Dingtao $\mathrm{Ma}^{1,2}$, Yiqing Shu ${ }^{2}$, Yuanjiang Xiang ${ }^{1}$, Jun Liu ${ }^{1}$, Jianqing $\mathrm{Li}^{2}$, \\ Krassimir Panajotov ${ }^{3}$, Yuwen Qin ${ }^{4}$ and Han Zhang ${ }^{1 *}$
}

The Kerr nonlinearity in two-dimensional (2D) nanomaterials is emerging as an appealing and intriguing research area due to their prominent light processing, modulation, and manipulation abilities. In this contribution, 2D black arsenicphosphorus (B-AsP) nanosheets (NSs) were applied in nonlinear photonic devices based on spatial self-phase modulation (SSPM) method. By applying the Kerr nonlinearity in 2D B-AsP, an all-optical phase-modulated system is proposed to realize the functions of "on" and "off" in all-optical switching. By using the same all-optical phase-modulated system, another optical logic gate is proposed, and the logical "or" function is obtained based on the 2D B-AsP NSs dispersions. Moreover, by using the SSPM method, a 2D B-AsP/SnS 2 hybrid structure is fabricated, and the result illustrates that the hybrid structure possesses the ability of the unidirectional nonlinear excitation, which helps in obtaining the function of spatial asymmetric light propagation. This function is considered an important prerequisite for the realization of diode functionalization, which is believed to be a factor in important basis for the design of isolators as well. The initial investigations indicate that 2D B-AsP is applicable for designing optical logical devices, which can be considered as an important development in all-optical information processing.

Keywords: B-AsP nanomaterial; all-optical phase-modulated system; spatial asymmetric light propagation

Wu LM, Fan TJ, Wei SR, Xu YJ, Zhang Y et al. All-optical logic devices based on black arsenic-phosphorus with strong nonlinear optical response and high stability. Opto-Electron Adv 5, 200046 (2022).

\section{Introduction}

The all-optical modulation technology is considered an emerging research field that may have a significant impact in the future. As compared to the conventional elec- trical modulation technology, the most prominent advantage of the all-optical modulation technology is its fast speed and strong anti-interference ability. Especially in a complex electromagnetic environment, the electrical

${ }^{1}$ Institute of Microscale Optoelectronics, Collaborative Innovation Centre for Optoelectronic Science \& Technology, Key Laboratory of Optoelectronic Devices and Systems of Ministry of Education and Guangdong Province, Shenzhen Key Laboratory of Micro-Nano Photonic Information Technology, Guangdong Laboratory of Artificial Intelligence and Digital Economy (SZ), Shenzhen University, Shenzhen 518060, China; ${ }^{2}$ Faculty of Information Technology, Macau University of Science and Technology, Macao 519020, China; ${ }^{3}$ Department of Applied Physics and Photonics (IR-TONA), Vrije Universiteit Brussels, Pleinlaan 2, B-1050 Brussels, Belgium; ${ }^{4}$ nstitute of Advanced Photonics Technology, School of Information Engineering, Guangdong University of Technology, Guangzhou 510006, China.

tThese authors contributed equally to this work.

"Correspondence: H Zhang, E-mail: hzhang@szu.edu.cn

Received: 7 August 2020; Accepted: 29 October 2020; Published online: 25 January 2022

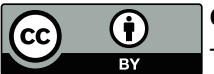

Open Access This article is licensed under a Creative Commons Attribution 4.0 International License.

(c) The Author(s) 2022. Published by Institute of Optics and Electronics, Chinese Academy of Sciences. 
modulation technology is considered more susceptible to interference, which adversely affects the modulation signal. In order to solve the deficiency of the traditional electro-optical and optoelectronic modulation technologies, the all-optical modulation technology ${ }^{1-4}$ is developed for designing new photonic devices ${ }^{5-8}$, so that information can always be processed in an optical approach, which thus ignores the disadvantages. The SSPM and spatial cross-phase modulation (SXPM) methods are considered interesting nonlinear optical phenomena, which are demonstrated to have a promising application in an all-optical technology 7,8 .

The SSPM and SXPM are considered the optical phenomena of the Kerr effect, which are developed from the nonlinear materials, and the 2D materials are considered the representative candidates. In recent years, the number of $2 \mathrm{D}$ atomic materials has been increasing, and the research methods have begun to diversify. The SSPM method is also developed as an effective method for studying the optical nonlinearity of new materials. Ji et al. studied the effect of gravity on the SSPM effect of carbon nanotubes'. Wu et al. used the SSPM for studying the optical nonlinear coherence behavior of graphene material over a wide band ${ }^{10}$. Wang et al. applied the SSPM to study the tunable method for the nonlinear effect of 2D graphene at a wavelength of $632.8 \mathrm{~nm}^{11}$. Moreover, the SXPM method based on two-color lasers is also proposed to apply in the all-optical switching. Zhao et al. innovatively used the $2 \mathrm{D} \mathrm{MoS}$ as an intermediate medium for the modulation of different lasers to achieve light-controlled light, which establishes the foundation for the application of all-optical switching based on SXPM method ${ }^{8}$. However, the applications of the SSPM and SXPM based on 2D materials are still in their infancies, and more investigations are urgently required to enrich the applications.

2D black phosphorus (BP), a fascinating 2D elemental material, has attracted adequate attention in the field of science and technology in recent years due to its remarkable physical and chemical properties. BP possesses high carrier mobility of $\sim 1,000 \mathrm{~cm}^{2} \mathrm{~V}^{-1} \mathrm{~s}^{-1}{ }^{12}$, a large "on/off" current ratio of $10^{4}{ }^{13}$, and a tunable band gap in the range of $(0.3 \mathrm{eV}-2.0 \mathrm{eV})^{14}$, which exhibits an appealing application potential in not only nanoelectronic devices, but also in nanophotonic devices such as random access memory ${ }^{15,16}$, all-optical signal processing ${ }^{17,18}$, phototherapy $^{19,20}$, solar cells ${ }^{21,22}$ and highly sensitive sensors ${ }^{23,24}$, etc. In addition, $\mathrm{BP}$ exhibits a strong nonlinear response so that its third-order nonlinear susceptibility is measured to be $\sim 10^{-8}$ (e.s.u.) based on the SSPM method ${ }^{25}$, and it can be applied in the all-optical technology ${ }^{17,26}$. However, BP demonstrates an unfavorable obstacle, namely, poor stability $^{27,28}$, in practical applications. In the air environment or the aqueous solution, BP easily reacts with oxygen, thereby weakens its photoelectric properties ${ }^{28,29}$. In order to resolve the instability defect of BP, many methods for improving stability have been reported in recent years, including metal-ion modification ${ }^{30}$, fluorination ${ }^{31}$, ligand surface coordination ${ }^{32}$, etc. Despite these promising developments, it is still challenging to simultaneously obtain high stability and retain performance similar to BP. Very recently, a substitute doping method, by adding some non-metallic atoms of As to BP to replace parts of the phosphorus atoms, has been proposed to improve the stability of $\mathrm{BP}$, and then the new material of $\mathrm{B}$ AsP is synthesized ${ }^{33}$. B-AsP, a derivative of phosphide 2D material, can be expressed in the form of $\mathrm{B}-\mathrm{As}_{x} \mathrm{P}_{1-x}$, where " $x$ " in the range of $0-0.83^{34}$. By varying the value of " $x$ ", the band gap of B-AsP can be modulated to have a broadband light -matter interaction. Moreover, 2D BAsP also possesses the properties of a fast photoresponse and a low dark noise in order to find an application in mid-wave infrared photoconductors ${ }^{34-36}$. However, the research on 2D B-AsP is still in its infancy, and there are many physical and chemical properties and applications that are required to be explored. Especially the optical nonlinearity in 2D B-AsP has not been studied so far. In this investigation, the nonlinear properties of $2 \mathrm{D}$ B-AsP are studied by applying the SSPM method to realize the device functions. B-AsP is demonstrated to have a stronger nonlinear optical response than the $\mathrm{BP}$, and a better optical stability as well. Considering the advantages of the high stability and the strong Kerr nonlinearity, the new 2D B-AsP material is well applied in the SSPM-based nonlinear switching, logical gate, and isolator.

\section{Fabrication and characterizations of 2D B-AsP}

The 2D B-AsP NSs used in our experiment were fabricated by using the $\mathrm{B}-\mathrm{As}_{x} \mathrm{P}_{1-x}$ crystals $(x=0.4)$ based on the method of liquid-phase exfoliation. First, $200 \mathrm{mg}$ of the B-AsP crystal was added to $200 \mathrm{~mL}$ of isopropanol (IPA), and a probe sonication with a power of $200 \mathrm{~mW}$ was used to exfoliate 2D B-AsP for 8 hours. Hereafter, the obtained B-AsP suspension was sonicated in a water bath overnight under the power of $400 \mathrm{~W}$, and the 
temperature is maintained to below $20^{\circ} \mathrm{C}$. After applying the probe sonication and the bath sonication successively, the resultant B-AsP suspension was then centrifuged at $3000 \mathrm{rpm}$ for 20 minutes. Finally, the supernatant of 2D B-AsP was extracted for further high-speed centrifugation (12000 rpm, $20 \mathrm{~min}$ ), and 2D B-AsP was used as eventually separated sediment in our experiments. The synthesis process of B-AsP crystals is discussed in Experimental section.

The resultant delaminated B-AsP was characterized by the scanning electron microscope (SEM) image, as shown in Fig. 1(a), which reveals a crystal structure of layered B-AsP nanomaterials. Figure 1(b) shows the orthorhombic crystal structure of B-AsP nanomaterials by the high-resolution TEM (HRTEM) image, and the interplanar spacing of lattice fringes for $\mathrm{B}$-AsP is observed to be $3.3 \AA$ and $4.7 \AA\left(1 \AA=10^{-10} \mathrm{~m}\right)$ in the zigzag and armchair directions, respectively. In the pattern of the selected area electron diffraction (SAED) (the inset image in Fig. 1(b)), the (100), (001) and (111) facets correspond to lattice constants of $3.3 \AA$, $4.7 \AA$ and $5.8 \AA$, which demonstrates the high crystalline quality of the fabricated 2D B-AsP. Figure 1(c) and 1(d) display an atomicforce microscopy (AFM) image of $2 \mathrm{D}$ B-AsP with a
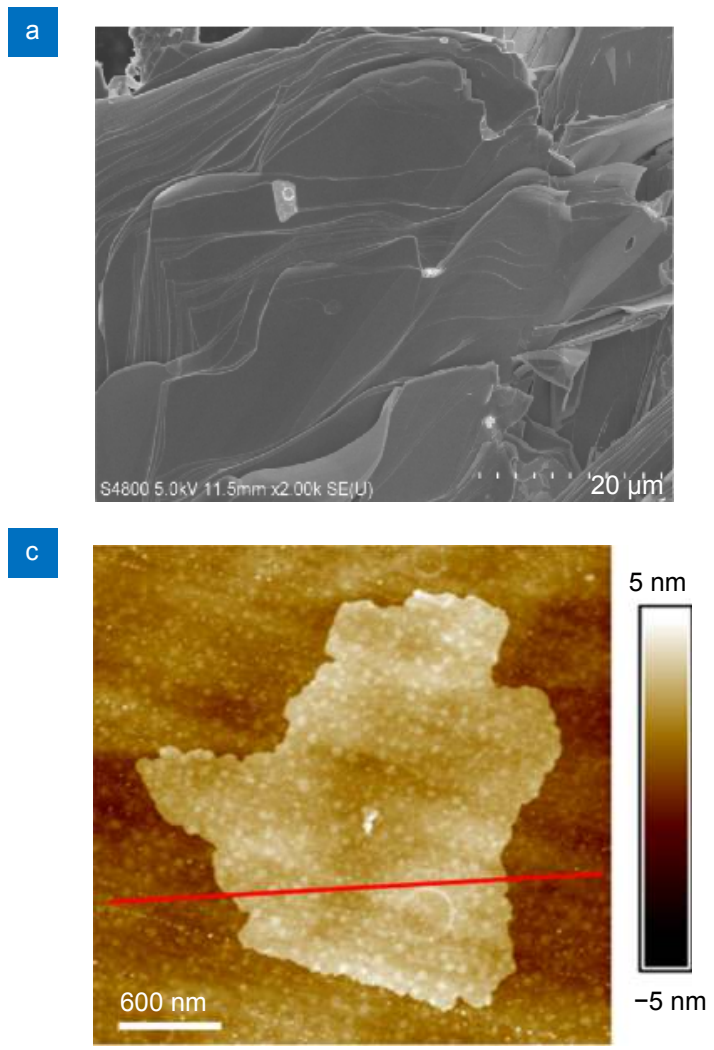

Fig. 1 (a) The SEM image of the layered B-AsP. (b) The high resolution TEM (HRTEM) image and the selected area electron diffraction (SAED) pattern of the 2D B-AsP. (c) The AFM image of 2D B-AsP. (d) The height profiles along the red line in (c). the $\mathrm{B}-\mathrm{As}_{x} \mathrm{P}_{1-x}$ nanomaterial was also investigated by using the first-principles approach (Fig. 2). When the value of " $x$ " is " 0 ", the direct band gap of $\mathrm{B}-\mathrm{As}_{0} \mathrm{P}_{1}$ is calculated to be $\approx 0.3 \mathrm{eV}$ at the $Z$ point (Fig. 2(a)), which well matches with previous theoretical and experimental investigations ${ }^{37-39}$. However, with the arsenic concentration increasing to a larger value, the band gap structure of the $\mathrm{B}-\mathrm{As}_{x} \mathrm{P}_{1-x}$ will be destroyed and modulated to a narrow value (Figure $2(b-d)$ ). It is worth noting that the $\mathrm{B}-\mathrm{As}_{x} \mathrm{P}_{1-x}$ exhibits the characteristic of a metal conductor when $x>0.4$ (Fig. 2(c) and 2(d)).

Calculations are performed in the density functional theory (DFT) framework as implemented in the Vienna ab initio simulation package (VASP) ${ }^{40}$. Exchange correlation energies were treated by using the generalized gradient approximation method with the Perdew-BurkeErnzerhof function ${ }^{41}$. The projector-augmented wave ${ }^{42}$ potential was used to describe the ion-electron interaction, and the plane wave cutoff energy was set as $450 \mathrm{eV}$. The Brillouin zone of the $k$-point meshes was set to $13 \times$ $9 \times 7,13 \times 9 \times 7,10 \times 7 \times 1,3 \times 7 \times 5$ for pure black-P, $\mathrm{As}_{0.25} \mathrm{P}_{0.75}, \mathrm{As}_{0.4} \mathrm{P}_{0.6}$ and $\mathrm{As}_{0.83} \mathrm{P}_{0.17}$, respectively. All structures are optimized until the force of each atom
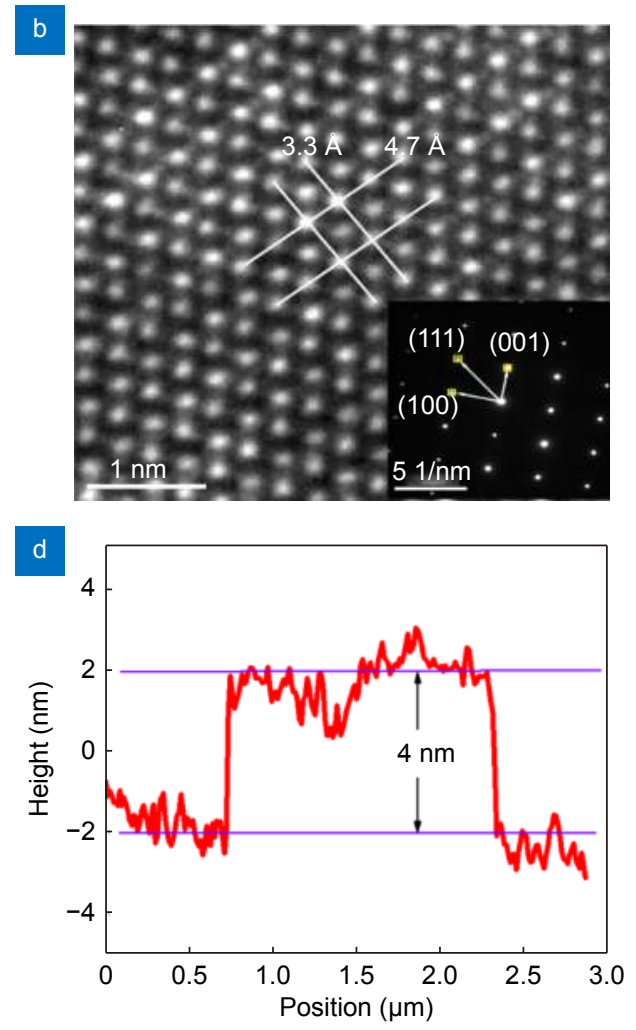

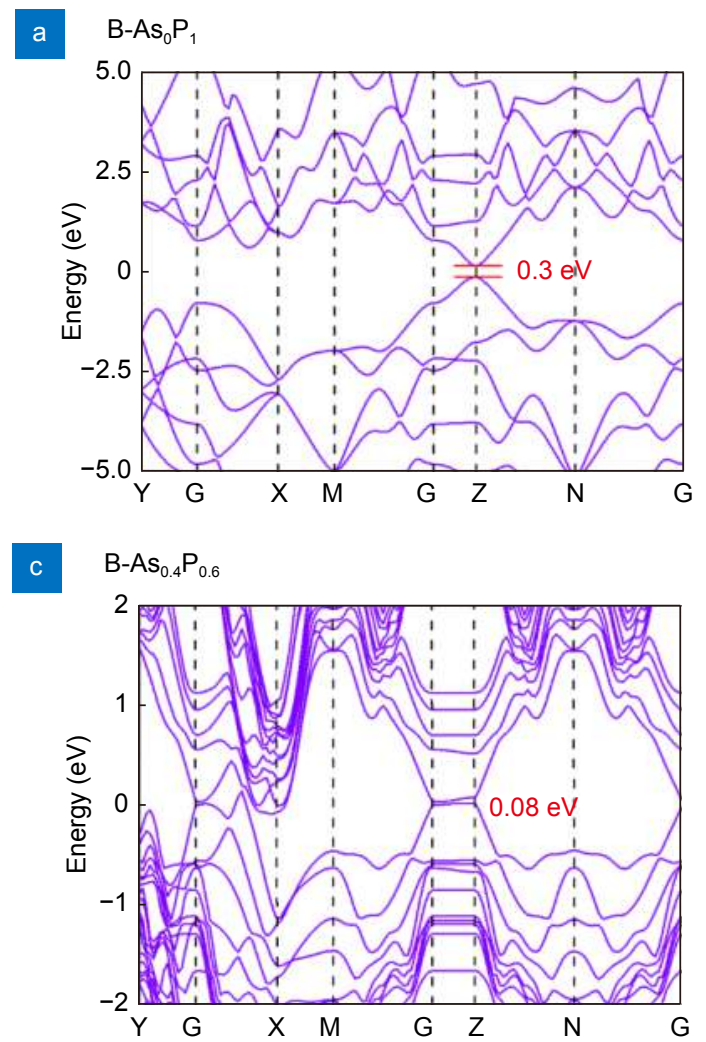

b

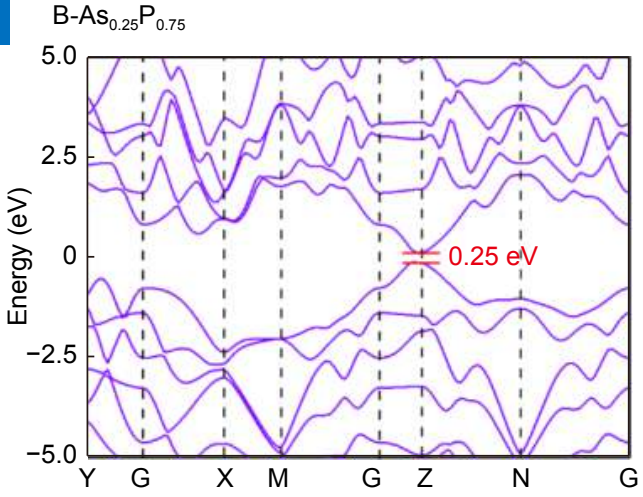

d

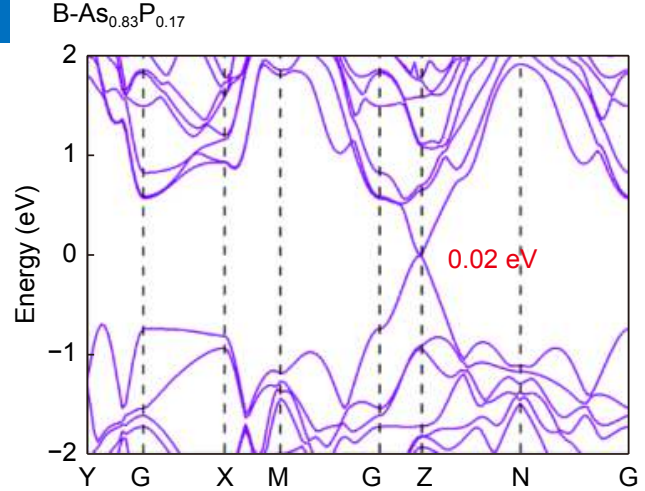

Fig. 2 | Band gap structures of the $B-A_{x} P_{1-x}$ with different value of " $x$ " ( $(x=0,0.25,0.4$, and 0.83$)$.

converged to $0.02 \mathrm{eV} / \AA$. The convergence criterion for energy was set as $10^{-5} \mathrm{eV}$ in relaxation and set as $10^{-6} \mathrm{eV}$ in the static calculation.

\section{Results and discussions}

\section{Nonlinear optical response of 2D B-AsP NSs}

Figure 2 shows the theoretical results of band gap structure of $\mathrm{B}-\mathrm{As}_{x} \mathrm{P}_{1-x}$ with different arsenic concentrations, and the result demonstrates that the band gap of $\mathrm{B}$ $\mathrm{As}_{x} \mathrm{P}_{1-x}(x=0.4)$ NSs used in our experiment is $0.08 \mathrm{eV}$ (Fig. 3(a)), which is similar to metals. In the following discussion, $\mathrm{B}-\mathrm{As}_{x} \mathrm{P}_{1-x}(x=0.4)$ is abbreviated as B-AsP. The light sources with the wavelengths of $\lambda=532 \mathrm{~nm}$ and $671 \mathrm{~nm}$ used in the experiment system are beyond the band gap, which indicates that the electrons in the valence band $(\mathrm{VB})$ can be excited into the conduction band (CB) (Fig. 3(d)) due to the good light-matter interaction in the B-AsP NSs dispersions. An obvious Tyndall effect is observed as the light sources transmit through the sample (Fig. 3(b)), and then the Kerr effect in the 2D B-AsP NSs can be excited if the intensity is strong enough. The incident laser beam is a Gaussian light, which exhibits the strongest intensity at the center, and then gradually weakens toward the surrounding.
The 2D B-AsP NSs are considered the intensity-dependent nonlinear materials so that the distribution of different intensities in the Gaussian spot will produce different phase-shift $(\Delta \psi)$ when the Gaussian light passes through the 2D B-AsP NSs, and the phase-shift distribution is considered a Gaussian-like curve (Fig. 3(c)). In the phase-shift curve, two points $\left(r_{1}\right.$ and $\left.r_{2}\right)$ with the same slope can be obtained, which illustrates that the phenomenon of interference will occur due to the same wave vector $\left((\mathrm{d} \Delta \psi / \mathrm{d} r)_{r 1}=(\mathrm{d} \Delta \psi / \mathrm{d} r)_{r 2}\right)$. The phase-shift of $r_{1}$ and $r_{2}$ meet the interference condition of $\Delta \psi\left(r_{1}\right)-$ $\Delta \psi\left(r_{2}\right)=M \pi$, where $M$ is an integer (odd or even), and hence the corresponding dark or bright stripes can be achieved, respectively. Hereafter, the pattern of diffraction rings is obtained on a black screen beyond the sample, which is the so-called SSPM ${ }^{43}$.

The diffraction patterns generated from the SSPM, which is caused by the Kerr nonlinearity in 2D B-AsP NSs, are sensitive to the intensity changes of incident laser beams. With the increasing incident intensity, a stronger nonlinear optical response will be excited to generate more diffraction rings (Fig. $3(\mathrm{e})$ and $3(\mathrm{f})$ ). The nonlinear optical response $(R)$ is defined as $R=N / I^{10,44}$, where $N$ and $I$ are the number of diffraction rings and the incident intensity, respectively. After the 

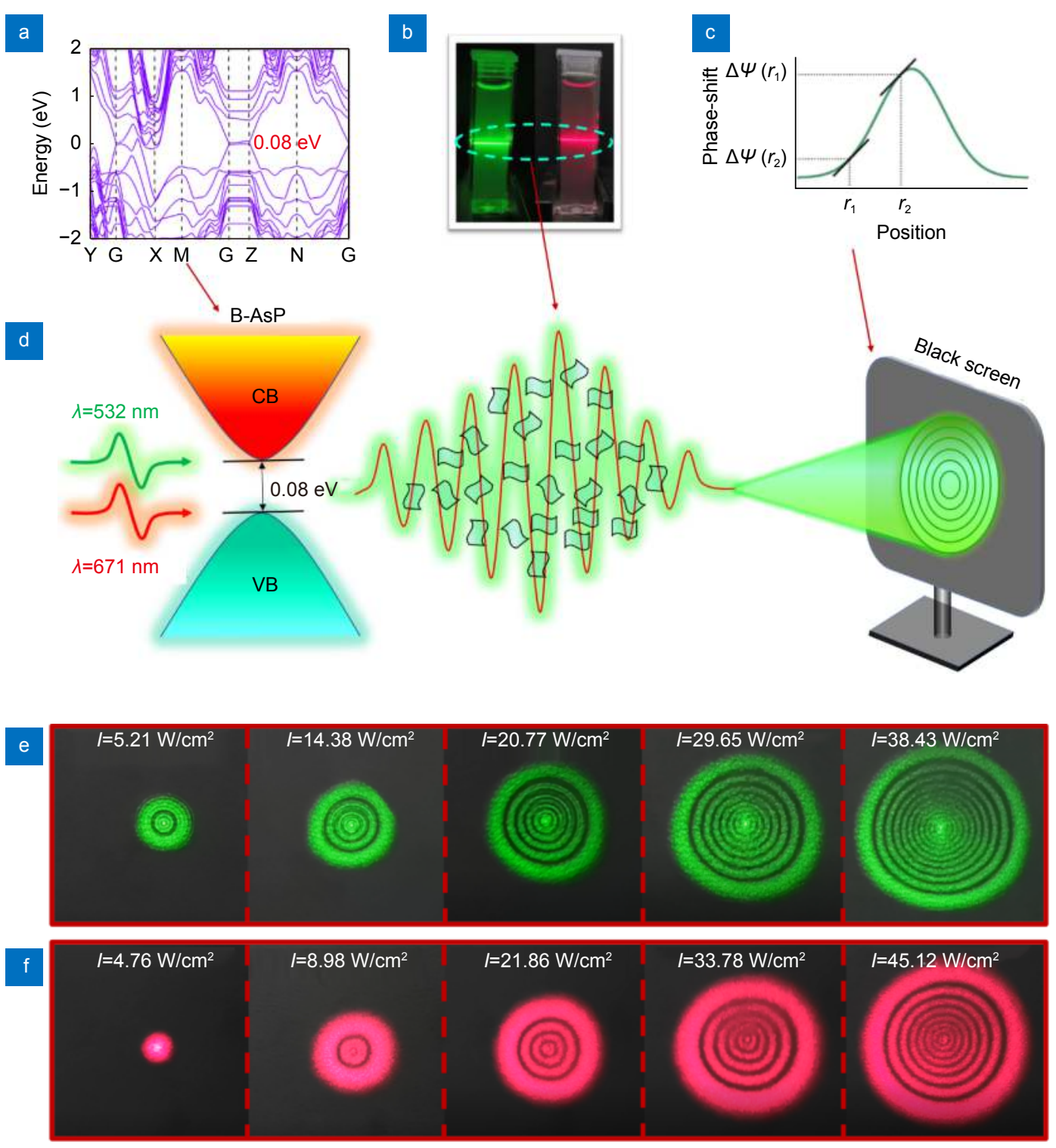

g

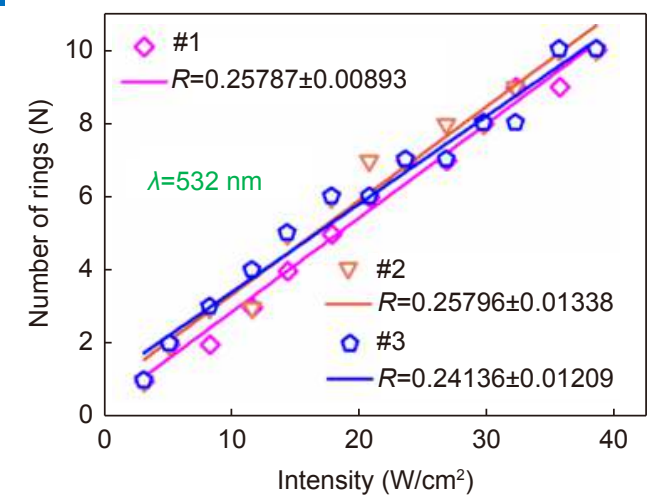

$\mathrm{h}$

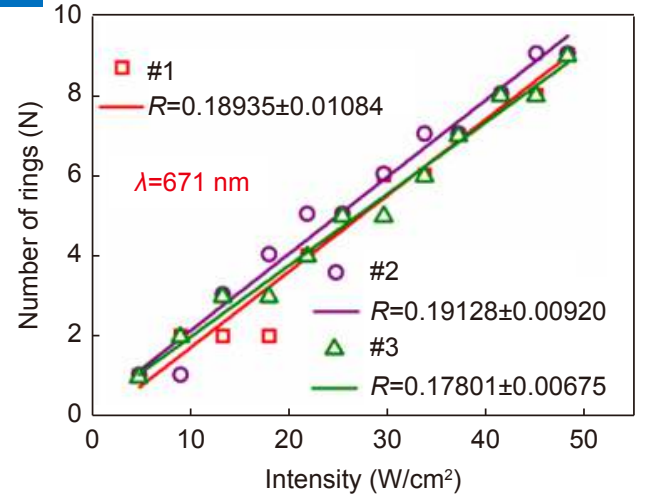

Fig. 3 | (a) The band gap structure of the B-As $\mathrm{P}_{1-x}(x=0.4)$ NSs. (b) The 2D B-AsP NSs dispersions used in our experiment, and the Tyndall effect observed as the laser beams transmitted through the sample. (c) The phase-shift of the incident light caused by the Kerr nonlinearity in $2 \mathrm{D}$ B-AsP NSs. (d) Experimental schematic of the SSPM based on the 2D B-AsP NSs dispersions. (e, f) The intensity-dependent diffraction patterns generated from the 2D B-AsP NSs dispersions with the lasers of $\lambda=532 \mathrm{~nm}$ and $671 \mathrm{~nm}$, respectively. $(\mathbf{g}, \mathbf{h})$ The nonlinear optical response $(R)$ for the 2D B-AsP NSs dispersions with three repeated measurements at $\lambda=532 \mathrm{~nm}$ and $671 \mathrm{~nm}$, respectively. 
measurement, the $R$ of 2D B-AsP is identified (Fig. 3(g) and $3(\mathrm{~h}))$. Herein, the $R$ of 2D B-AsP NSs dispersions $(0.0025 \mathrm{mg} / \mathrm{mL})$ is measured in triplicate at $\lambda=532 \mathrm{~nm}$ and $671 \mathrm{~nm}$, respectively. The results demonstrate that the $R$ of the sample at $\lambda=532 \mathrm{~nm}$ and $671 \mathrm{~nm}$ is approximately equal to 0.25 and 0.18 , respectively, with high repeatability. Moreover, the $R$ of the B-AsP material also has a dependence on concentration. When the concentrations of B-AsP are $C_{1}=0.0025 \mathrm{mg} / \mathrm{mL}$ and $C_{2}=$ $0.0050 \mathrm{mg} / \mathrm{mL}$, the corresponding values of $R$ are 0.258 $\mathrm{cm}^{2} / \mathrm{W}$ and $0.501 \mathrm{~cm}^{2} / \mathrm{W}$, which demonstrates that after the concentration of the B-AsP material is doubled, the corresponding nonlinear response is also nearly doubled accordingly (Fig. S1 of the Supplementary information).

The optical stability of the 2D B-AsP NSs dispersions are also be characterized in Fig. 4. A strong enough 671 $\mathrm{nm}$ laser beam with a fixed intensity passes through the sample for a consecutive 6 hours, and the changes of diffraction rings are recorded in real time by a camera. The diffraction patterns are shown in Fig. 4(a) for every hour, which illustrates that the 2D B-AsP NSs dispersions possess a good optical stability under the exposure of strong incident light. The absorption spectra of the 2D B-AsP NSs dispersions before and after 6 hours of exposure are shown in Fig. 4(b). Only a slight change in absorption spectra is measured, which hardly affects the experimental results. The nonlinear optical response of BP and B-AsP is shown in Figure S2 of the Supporting Information. The nonlinear refractive index and the third-order nonlinear susceptibility of B-AsP can be obtained as: $n_{2}=\frac{\lambda}{2 n_{0} L_{\mathrm{eff}}} \cdot \frac{N}{I}$, and $\chi_{\text {monolayer }}^{(3)}=\frac{c n_{0} \lambda}{2.4 \times 10^{4} \pi^{2} L_{\mathrm{eff}} N_{\mathrm{eff}}^{2}} \cdot \frac{N}{I}$

a

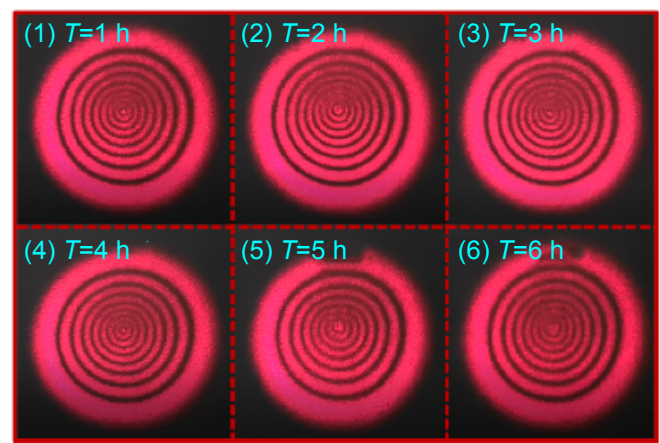

(the $\lambda, n_{0}$, and $L_{\text {eff }}$ are the wavelength, linear refractive index, and effective optical thickness of materials) ${ }^{8}$, which indicates that the $n_{2}$ and $\chi^{(3)}$ are proportional to $N / I$ (nonlinear optical response). Previous report ${ }^{25}$ indicates that the nonlinear refractive index and the third-order nonlinear susceptibility of BP at the wavelength range of $350-1160 \mathrm{~nm}$ are measured to be $n_{2} \sim 10^{-5}$ $\mathrm{cm}^{2} / \mathrm{W}$ and $\chi^{(3)} \sim 10^{-8}$. In this work, the nonlinear optical response of $2 \mathrm{D} \mathrm{B}-\mathrm{AsP}$ is measured and compared with BP (Fig. S2 of the Supplementary information). The result indicates that the nonlinear optical response of $\mathrm{B}$-AsP is slightly larger than that of $\mathrm{BP}$, and this means that the $\chi^{(3)}$ of B-AsP is approximately $\sim 10^{-8}$, similar to BP.

\section{D B-AsP-based all-optical phase modulated system}

By using the Kerr nonlinearity in the 2D B-AsP, a 2D BAsP-based all-optical phase-modulated system (Fig. 5(a)) is proposed based on the SXPM method to realize lightto-light modulation. Normally, when two beams of light meet in space, they do not interact with each other, and continue to propagate along their respective directions. However, this phenomenon fails if the lights meet in the 2D B-AsP NSs dispersions. The nonlinear material of 2D B-AsP NSs has the ability to modulate the phase of incident lights. When the incident lights are beyond the band gap of 2D B-AsP NSs and their intensities are strong enough, the SSPM will be excited. The SXPM refers to the mutual modulation of the phase between two laser beams, including a controlling light and the other signal light. First of all, the $532 \mathrm{~nm}$ green laser beam is selected as the signal light, which is modulated to a low intensity $\left(<4 \mathrm{~W} / \mathrm{cm}^{2}\right)$, which is well below the excitation threshold

b

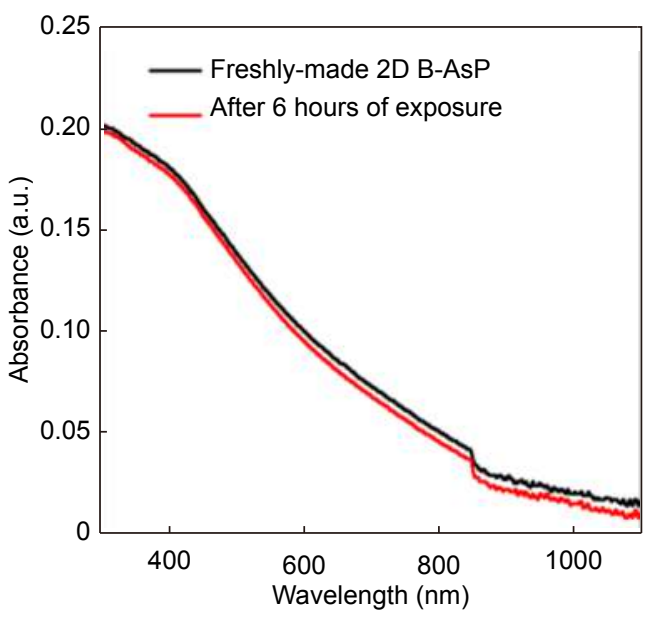

Fig. 4 | (a) The diffraction patterns excited from the 2D B-AsP NSs dispersions with a high light intensity for consecutive 6 hours. (b) The absorption spectrums for the 2D B-AsP NSs dispersions before and after 6 hours of exposure. 


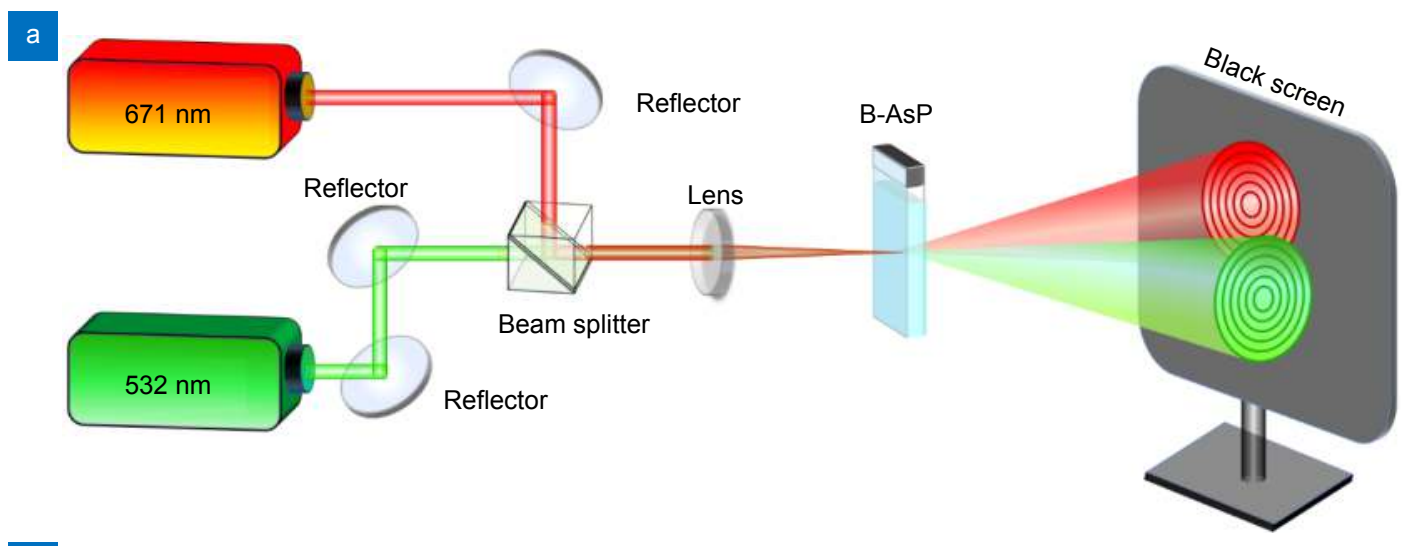

b

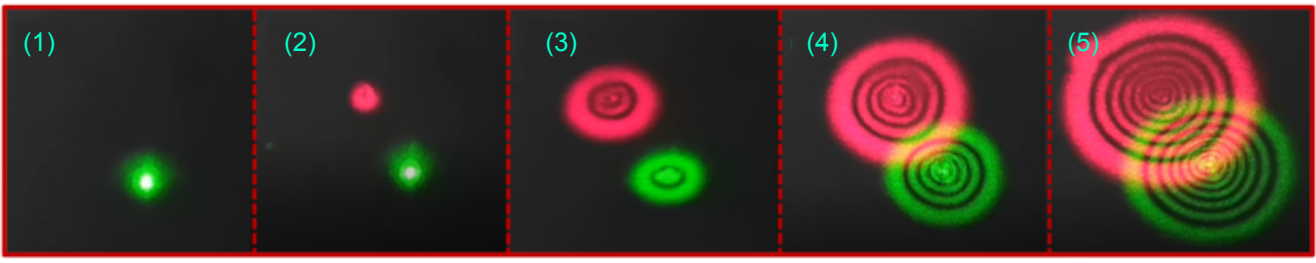

Increasing the incident intensity of red light

c

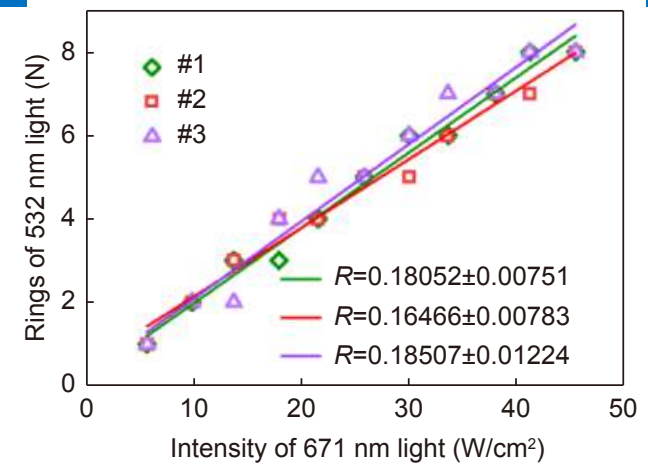

d

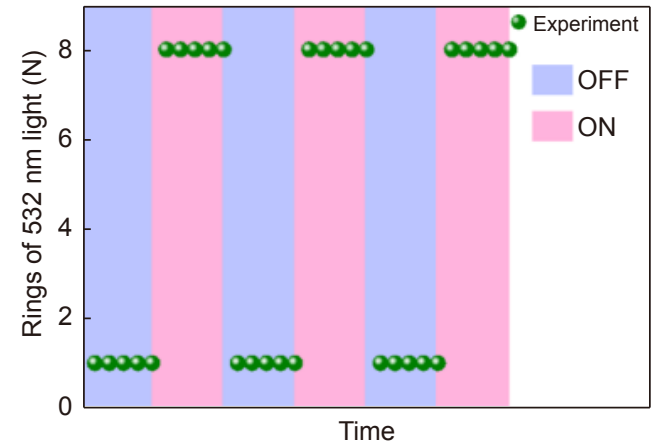

e

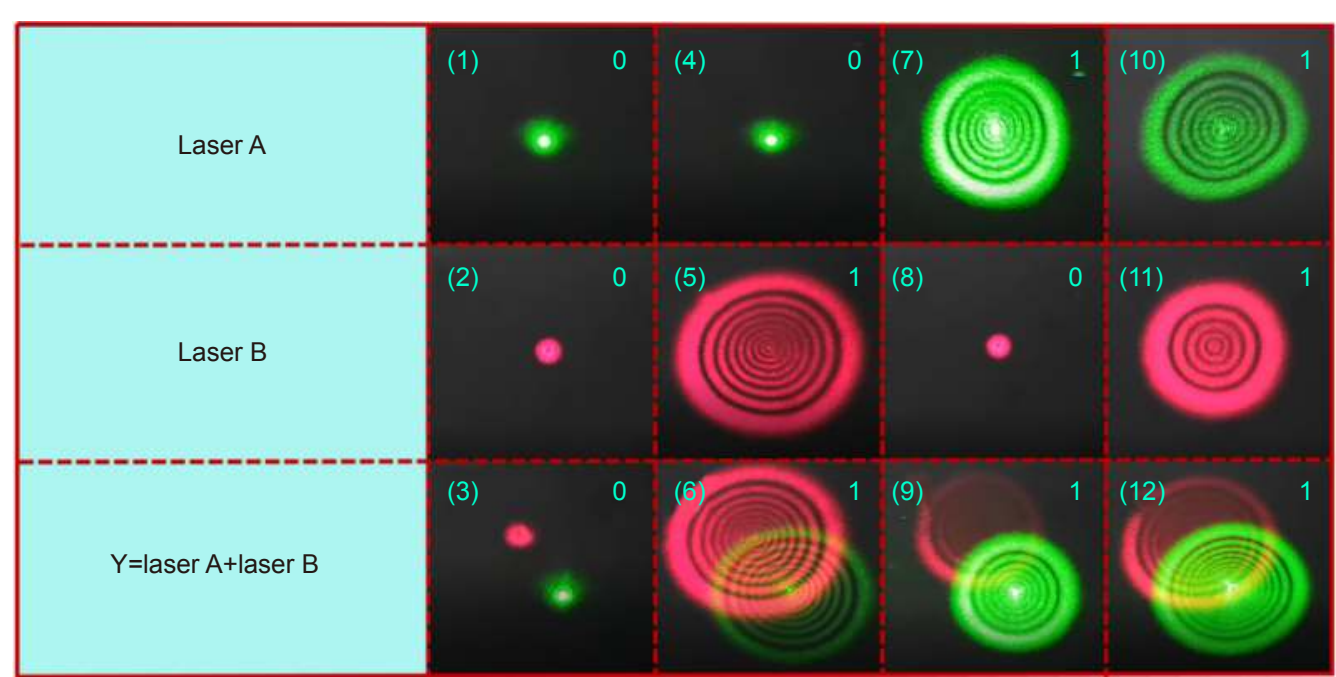

Fig. 5 | (a) Experimental schematic of the 2D B-AsP-based all-optical phase modulated system. (b) The phase modulation of controlling light to signal light. (c) The number of diffraction rings modulated by the intensity of controlling light. (d) Result of the all-optical switching based on 2D BAsP NSs to realize the functions of "on" and "off". (e) 2D B-AsP NSs all-optical logical gate to achieve the "or" function. 
of the nonlinear optical response (Fig. 5(b-1)). Therefore, the other controlling light (671 nm red light) with strong enough intensity is incorporated into the optical path by using a beam splitter (BS), and then the diffraction rings of the controlling light are excited. More interestingly, the diffraction pattern of the signal light (green light) is also induced, and the number of diffraction rings is increased with an increase in the controlling light intensity (Fig. 5(b)). When the controlling light passes through the sample, the refractive index of B-AsP NSs changes due to the Kerr effect, which then inversely modulates the phase of the incident light. According to the SXPM method, the light field of the signal light will be promoted by the controlling light in order to enhance the phase-shift, which further generates the diffraction rings.

The number of diffraction rings of the signal light $(\lambda=$ $532 \mathrm{~nm}$ ) modulated by the intensity of controlling light $(\lambda=671 \mathrm{~nm})$ is shown in Fig. 5(c). The $R$ of the signal light is found to be approximately equal to 0.18 , which similar to the $R$ of the $671 \mathrm{~nm}$ laser beam measured in Fig. 3(h). The 2D B-AsP-based all-optical phase-modulated system exhibits good application prospect in the all-optical switching. In the modulated system, both the signal light and controlling light are set to a fixed intensity so that the controlling light has a strong intensity $\left(45.32 \mathrm{~W} / \mathrm{cm}^{2}\right)$, whereas the intensity of the signal light has a low value $\left(3.76 \mathrm{~W} / \mathrm{cm}^{2}\right)$. When the controlling light is set to the "ON" mode, the number of diffraction rings of the signal light will be induced to be 8 ; when the controlling light is set to the "OFF" mode, the number of diffraction rings of the signal light will be induced to be 1 (Fig. 5(d)). The "excitation" and the "non-excitation" of the diffraction rings in the signal light can be considered as the "on" and "off" instructions in the B-AsP-based alloptical switching.

Moreover, taking advantage of the all-optical phasemodulated system, another 2D B-AsP-based all-optical logical gate is proposed to realize the function of "or" gate. The "or" gate possesses four sub-functions, namely, " $0+0=0$ ", " $0+1=1$ ", " $1+0=1$ ", and " $1+1=1$ ". Normally, the "or" gate is implemented by electricity, and here we have proposed an all-optical pathway of using 2D B-AsP to realize the logical function of the "or" gate. In our all-optical phase-modulated system, "Laser A" and "Laser B" are the input signals, and "Y = Laser A + Laser B" is the output signal. The "excitation" and the "non-excitation" of the diffraction rings are represented by the output signals in a high level of "1" and a low level of "0", respectively. First, both "Laser A" and "Laser B" are modulated below the threshold so that the diffraction rings cannot be excited, and the inputs are two lowlevel signals of " 0 " (Fig. 5(e)-(1,2)). By using the 2D BAsP-based all-optical phase modulated system, these two input signals are processed, and the output signal $(\mathrm{Y})$ is also at a low level of "0" (Fig. 5(e)-(3)). Second, if the "Laser A" is set at a low level of "0", whereas "Laser B" is set at a high level of " 1 ", the output signal will become a high level of "1" (Fig. 5(e)-(4-6)). Third, if "Laser A" and "Laser B" are set at a high level of " 1 " and a low level of "0", respectively, the output signal of "Y" will reveal a high level of "1" (Fig. 5(e)-(7-9)). Form Fig. 5(e)-(4-6) and Fig. 5(e)-(7-9), it can be observed that the diffraction rings excited by different wavelengths can be modulated with each other. Fourth, when both "Laser A" and "Laser B" are capable of generating the diffraction rings in a high level of "1", the output signal will still maintain a high level of "1" (Fig. 5(e)-(10-12)).

\section{Spatial asymmetric light propagation in 2D B-AsP}

In this section, we describe a hybrid structure that enables spatial asymmetric light propagation (namely nonreciprocal light propagation). 2D B-AsP is demonstrated to possess a strong nonlinear optical response with a narrow band gap of $0.08 \mathrm{eV}$ (Fig. 3(a)), which displays a potential application in photonic devices. In order to realize the ability of spatial asymmetric light propagation in a photonic diode or an isolator, an auxiliary material of $2 \mathrm{D} \mathrm{SnS}_{2}$ is introduced and combined with $2 \mathrm{D} \mathrm{B}-\mathrm{AsP}$ to form a hybrid structure. $2 \mathrm{D} \mathrm{SnS}_{2}$ is a reverse saturable absorption (RSA) material ${ }^{45}$, which possesses a large band gap $\left(E_{\mathrm{g}}=2.6 \mathrm{eV}\right)^{46}$. The light sources used in our experiment are within the band gap of $\mathrm{SnS}_{2}$ so that the ability of light-matter interaction requires two-photon absorption to achieve the excitation of electrons at the VB into the CB. In the experiment of the SSPM, the Kerr effect of the 2D material is used instead of the saturated absorption effect. For the excitation of the Kerr nonlinearity in 2D B-AsP only a relatively low intensity is required, and the low intensity is found not strong enough to support the two-photon absorption behavior in $2 \mathrm{D}$ $\mathrm{SnS}_{2}$. When the laser beams pass through $\mathrm{SnS}_{2}$ dispersions, the light-matter interaction does not occur so that the diffraction rings cannot be excited.

Herein, the 2D B-AsP and $\mathrm{SnS}_{2}$ dispersions are respectively filled in two quartz cuvettes that are closed together to form a hybrid structure (Fig. 6). When a laser beam $(\lambda=671 \mathrm{~nm})$ passes through in the positive 


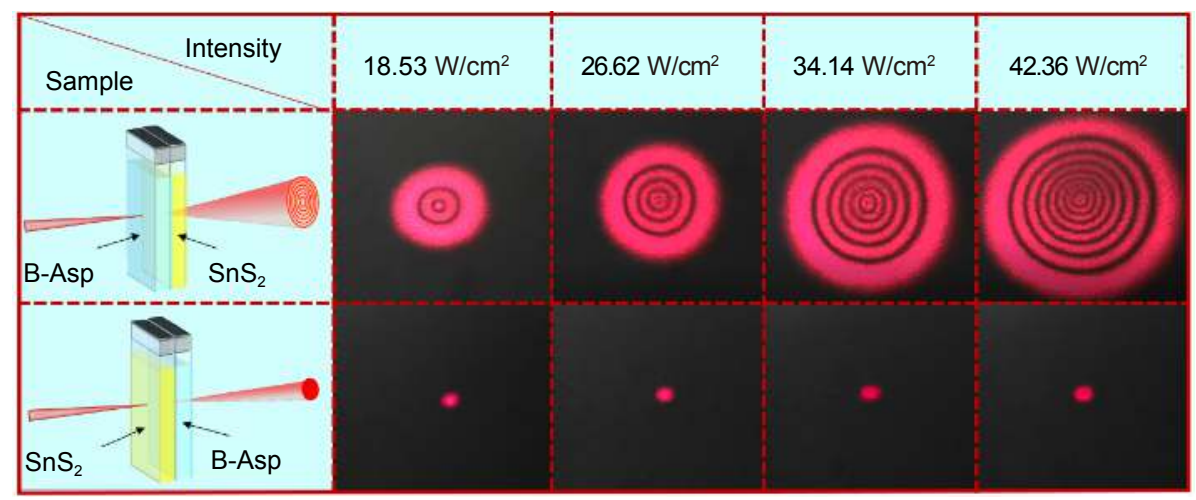

Fig. 6 | The unidirectional nonlinear excitation in 2D B-AsP/ $\mathrm{SnS}_{2}$ hybrid structure to achieve the spatial asymmetric light propagation.

direction (B-AsP/ $\left.\mathrm{SnS}_{2}\right)$, the light first interacts with the sample of 2D B-AsP NSs to excite the Kerr effect and further generate the diffraction rings. Thereafter, the light spot forming the diffraction rings will continue to pass through the second part of the $2 \mathrm{D} \mathrm{SnS}_{2}$ material. In $2 \mathrm{D} \mathrm{SnS}_{2}$ dispersions, the number of rings does not change since there is no interaction between light and $\mathrm{SnS}_{2}$, but the light intensity is weakened due to the linear absorption. Finally, we can observe that the diffraction rings are generated in a positive direction. Conversely, if the laser beam passes through in the opposite direction $\left(\mathrm{SnS}_{2} / \mathrm{B}-\mathrm{AsP}\right)$, the light first interacts with the $\mathrm{SnS}_{2}$ material, but the diffraction rings cannot be excited and the intensity is weakened by the linear absorption. Thereafter, the weakened light continues to pass through the sample of B-AsP dispersions. However, the light intensity is seriously weakened, which is found to be lower than the threshold of the excited Kerr nonlinearity in 2D $\mathrm{B}-\mathrm{AsP}$, and the diffraction rings cannot be generated, hence only a light point can be obtained from the opposite direction. The unidirectional nonlinear excitation in the $2 \mathrm{D} \mathrm{B}-\mathrm{AsP} / \mathrm{SnS}_{2}$ hybrid structure is considered an important prerequisite for the realization of diode functionalization as well as the design of isolators in order to obtain the spatial asymmetric light propagation. This phenomenon of the unidirectional nonlinear excitation can be obtained not only at $\lambda=671 \mathrm{~nm}$, but also at $\lambda=$ $532 \mathrm{~nm}$ (Figure S3, Supplementary information), which indicates that the hybrid structure shows a broadband optical response to realize the spatial asymmetric light propagation.

\section{Conclusion}

In this work, we have successfully fabricated a new $2 \mathrm{D}$ material, namely, 2D B-AsP NSs, the band gap of which is calculated to be $0.08 \mathrm{eV}$. In addition, the morphology, size, and lattice distance of the 2D B-AsP NSs are also characterized. Thereafter, we have explored the nonlinear optical properties of the B-AsP nanomaterial and applied it in the design of photonic devices. First, by using the SSPM method, the nonlinear optical response of 2D B-AsP NSs is measured in a broadband with a high optical stability. Second, a 2D B-AsP-based all-optical phase-modulated system based on the SXPM method is proposed in order to obtain the modulation of the controlling light to the signal light. Depending on the phasemodulated system, an all-optical switching is designed to realize the functions of "on" and "off" based on the Kerr nonlinearity in 2D B-AsP. Moreover, based on the same phase-modulated system, another optical logical gate that possesses the "or" function is proposed. Third, a hybrid structure consisting of $2 \mathrm{D} \mathrm{B}-\mathrm{AsP}$ and $\mathrm{SnS}_{2}$ is explored by using the SSPM method, and the results demonstrate that the hybrid structure is able to generate the effect of the unidirectional nonlinear excitation, breaking the spatial light reversible propagation, which provides a feasible solution to the design of the photonic diodes or the optical isolators.

\section{Experimental section}

The mineralizer-assisted chemical vapor transport (CVT) method was used to fabricate high-quality B-AsP. The precursors used in the experiment were gray arsenic and red phosphorus (molar ratio, $4: 6$ ), and the total mass was $500 \mathrm{mg}$. The precursor and the $10 \mathrm{mg}$ mineralization agent (tin iodide, $\mathrm{SnI}_{4}$ ) were placed horizontally in a vacuum quartz tube and sealed by the Partulab device (MRVS-1002). The furnace was heated to $750{ }^{\circ} \mathrm{C}$ at a rate of $5{ }^{\circ} \mathrm{C} / \mathrm{min}$ and maintained for 2 hours. Then, the furnace was cooled to $500{ }^{\circ} \mathrm{C}$ for 7.5 hours and maintained for 3 hours. After slowly cooling to $150{ }^{\circ} \mathrm{C}$ for 8 hours, the furnace was cooled to room temperature. Then the B-AsP crystals can be obtained at the cold end 
of the tube.

The scanning electron microscope (SEM, Quanta FEG 250) was used to investigate the morphology of B-AsP NSs. The transmission electron microscope (TEM) image and the SAED pattern were obtained from a Tecnai G2 F20 S-TWIN (field-emission) scanning transmission electron microscope operating at $200 \mathrm{kV}$. Atomic-force microscopy (AFM, BRUKER Dimension ICON, 512 pixels per line) was used to determine the thickness and the morphology of 2D B-AsP. The sample for the AFM measurement was prepared by dispersing $2 \mathrm{D}$ B-AsP into IPA and dropping it onto $\mathrm{Si} / \mathrm{SiO}_{2}$ substrates.

\section{References}

1. Paredes-Barato D, Adams CS. All-optical quantum information processing using rydberg gates. Phys Rev Lett 112, 040501 (2014).

2. Larger L, Soriano MC, Brunner D, Appeltant L, Gutierrez JM et al. Photonic information processing beyond Turing: an optoelectronic implementation of reservoir computing. Opt Express 20, 3241-3249 (2012).

3. Wang C, Chen QY, Chen HL, Liu J, Song YF et al. Boron quantum dots all-optical modulator based on efficient photothermal effect. Opto-Electron Adv 4, 200032 (2021).

4. Zheng JL, Tang $X$, Yang ZH, Liang ZM, Chen YX et al. Few-layer phosphorene-decorated microfiber for all-optical thresholding and optical modulation. Adv Opt Mater 5, 1700026 (2017).

5. Wu LM, Huang WC, Wang YZ, Zhao JL, Ma DT et al. 2D tellurium based high-performance all-optical nonlinear photonic devices. Adv Funct Mater 29, 1806346 (2019).

6. Liao K, Chen Y, Yu ZC, Hu XY, Wang XY et al. All-optical computing based on convolutional neural networks. Opto-Electron Adv 4, 200060 (2021).

7. Lu L, Wang WH, Wu LM, Jiang XT, Xiang YJ et al. All-optical switching of two continuous waves in few layer bismuthene based on spatial cross-phase modulation. ACS Photonics 4, 2852-2861 (2017).

8. Wu YL, Wu Q, Sun F, Cheng $C$, Meng $S$ et al. Emergence of electron coherence and two-color all-optical switching in $\mathrm{MoS}_{2}$ based on spatial self-phase modulation. Proc Natl Acad Sci USA 112, 11800-11805 (2015).

9. Ji W, Chen WZ, Lim S, Lin JY, Guo ZX. Gravitation-dependent, thermally-induced self-diffraction in carbon nanotube solutions. Opt Express 14, 8958-8966 (2006).

10. Wu R, Zhang YL, Yan SC, Bian F, Wang WL et al. Purely coherent nonlinear optical response in solution dispersions of graphene sheets. Nano Lett 11, 5159-5164 (2011).

11. Wang GZ, Zhang SF, Umran FA, Cheng X, Dong NN et al. Tunable effective nonlinear refractive index of graphene dispersions during the distortion of spatial self-phase modulation. Appl Phys Lett 104, 141909 (2014).

12. Li LK, Yu YJ, Ye GJ, Ge QQ, Ou XD et al. Black phosphorus field-effect transistors. Nat Nanotechno/ 9, 372-377 (2014).

13. Liu H, Neal AT, Zhu Z, Luo Z, Xu XF et al. Phosphorene: an unexplored 2D semiconductor with a high hole mobility. ACS Nano 8, 4033-4041 (2014).

14. Liu H, Du YC, Deng YX, Ye PD. Semiconducting black phos- phorus: synthesis, transport properties and electronic applications. Chem Soc Rev 44, 2732-2743 (2015).

15. Hao CX, Wen FS, Xiang JY, Yuan SJ, Yang BC et al. Liquid-exfoliated black phosphorous nanosheet thin films for flexible resistive random access memory applications. Adv Funct Mater 26, 2016-2024 (2016).

16. Han ST, Hu L, Wang XD, Zhou Y, Zeng YJ et al. Black phosphorus quantum dots with tunable memory properties and multilevel resistive switching characteristics. Adv Sci 4, 1600435 (2017).

17. Zheng JL, Yang ZH, Si C, Liang ZM, Chen X et al. Black phosphorus based all-optical-signal-processing: toward high performances and enhanced stability. ACS Photonics 4, 1466-1476 (2017).

18. Wang K, Chen YX, Zheng JL, Ge YQ, Ji JH et al. Black phosphorus quantum dot based all-optical signal processing: ultrafast optical switching and wavelength converting. Nanotechnology 30, 415202 (2019).

19. Qiu M, Wang D, Liang WY, Liu LP, Zhang $Y$ et al. Novel concept of the smart NIR-light-controlled drug release of black phosphorus nanostructure for cancer therapy. Proc Natl Acad Sci USA 115, 501-506 (2018).

20. Tao W, Zhu XB, Yu XH, Zeng XW, Xiao QL et al. Black phosphorus nanosheets as a robust delivery platform for cancer theranostics. Adv Mater 29, 1603276 (2017).

21. Hu W, Lin L, Yang C, Dai J, Yang JL. Edge-modified phosphorene nanoflake heterojunctions as highly efficient solar cells. Nano Lett 16, 1675-1682 (2016).

22. Dai J, Zeng XC. Bilayer phosphorene: effect of stacking order on bandgap and its potential applications in thin-film solar cells. J Phys Chem Lett 5, 1289-1293 (2014).

23. Abbas AN, Liu BL, Chen L, Ma YQ, Cong $S$ et al. Black phosphorus gas sensors. ACS Nano 9, 5618-5624 (2015).

24. Wu LM, Guo J, Wang QK, Lu SB, Dai XY et al. Sensitivity enhancement by using few-layer black phosphorus-graphene/TMDCs heterostructure in surface plasmon resonance biochemical sensor. Sens Actuators B Chem 249, 542-548 (2017).

25. Zhang JD, Yu XF, Han WJ, Lv BS, Li XH et al. Broadband spatial self-phase modulation of black phosphorous. Opt Lett 41, 1704-1707 (2016).

26. Xu YH, Wang WX, Ge YQ, Guo HY, Zhang XJ et al. Stabilization of black phosphorous quantum dots in pmma nanofiber film and broadband nonlinear optics and ultrafast photonics application. Adv Funct Mater 27, 1702437 (2017).

27. Doganov RA, O'Farrell ECT, Koenig SP, Yeo YT, Ziletti A et al. Transport properties of pristine few-layer black phosphorus by van der Waals passivation in an inert atmosphere. Nat Commun 6, 6647 (2015).

28. Favron A, Gaufrès E, Fossard F, Phaneuf-L'Heureux AL, Tang NYW et al. Photooxidation and quantum confinement effects in exfoliated black phosphorus. Nat Mater 14, 826-832 (2015).

29. Wood JD, Wells SA, Jariwala D, Chen KS, Cho E et al. Effective passivation of exfoliated black phosphorus transistors against ambient degradation. Nano Lett 14, 6964-6970 (2014).

30. Guo ZN, Chen S, Wang ZZ, Yang ZY, Liu F et al. Metal-ionmodified black phosphorus with enhanced stability and transistor performance. Adv Mater 29, 1703811 (2017).

31. Tang X, Liang WY, Zhao JL, Li ZJ, Qiu M et al. Fluorinated phosphorene: electrochemical synthesis, atomistic fluorination, and enhanced stability. Small 13, 1702739 (2017). 
32. Zhao $\mathrm{YT}$, Wang HY, Huang $\mathrm{H}$, Xiao QL, Xu YH et al. Surface coordination of black phosphorus for robust air and water stability. Angew Chem Int Ed 55, 5003-5007 (2016).

33. Liu BL, Köpf M, Abbas AN, Wang XM, Guo QS et al. Black arsenic-phosphorus: layered anisotropic infrared semiconductors with highly tunable compositions and properties. Adv Mater 27, 4423-4429 (2015).

34. Long MS, Gao AY, Wang P, Xia H, Ott C et al. Room temperature high-detectivity mid-infrared photodetectors based on black arsenic phosphorus. Sci Adv 3, e1700589 (2017).

35. Yuan SF, Shen CF, Deng BC, Chen XL, Guo QS et al. Airstable room-temperature mid-infrared photodetectors based on hBN/Black arsenic phosphorus/hBN heterostructures. Nano Lett 18, 3172-3179 (2018).

36. Amani M, Regan E, Bullock J, Ahn GH, Javey A. Mid-wave infrared photoconductors based on black phosphorus-arsenic alloys. ACS Nano 11, 11724-11731 (2017).

37. Qiao JS, Kong XH, Hu ZX, Yang F, Ji W. High-mobility transport anisotropy and linear dichroism in few-layer black phosphorus. Nat Commun 5, 4475 (2014).

38. Keyes RW. The electrical properties of black phosphorus. Phys Rev 92, 580-584 (1953).

39. Warschauer D. Electrical and optical properties of crystalline black phosphorus. J Appl Phys 34, 1853-1860 (1963).

40. Kresse G, Furthmüller J. Efficient iterative schemes for ab initio total-energy calculations using a plane-wave basis set. Phys Rev B 54, 11169-11186 (1996).

41. Perdew JP, Burke K, Ernzerhof M. Generalized gradient approximation made simple. Phys Rev Lett 77, 3865-3868 (1996).

42. Kresse G, Joubert D. From ultrasoft pseudopotentials to the projector augmented-wave method. Phys Rev B 59, 17581775 (1999).
43. Durbin SD, Arakelian SM, Shen YR. Laser-induced diffraction rings from a nematic-liquid-crystal film. Opt Lett 6, 411-413 (1981).

44. Wu LM, Dong YZ, Zhao JL, Ma DT, Huang WC et al. Kerr nonlinearity in $2 \mathrm{D}$ graphdiyne for passive photonic diodes. Adv Mater 31, 1807981 (2019).

45. Wu JJ, Tao YR, Wu XC, Chun Y. Nonlinear optical absorption of $\mathrm{SnX}_{2}(\mathrm{X}=\mathrm{S}$, Se) semiconductor nanosheets. J Alloys Compd 713, 38-45 (2017).

46. Ye GL, Gong YJ, Lei SD, He YM, Li B et al. Synthesis of largescale atomic-layer $\mathrm{SnS}_{2}$ through chemical vapor deposition. Nano Res 10, 2386-2394 (2017).

\section{Acknowledgements}

Financial supports from the National Natural Science Foundation of China (NSFC) (61435010 and 21773168), the Science and Technique Planning Project of Guangdong Province (Grant No. 2016B050501005), the Science and Technology Innovation Commission of Shenzhen (JCYJ20170302153323978 and JCYJ201704101719588539), the Science and Technology Development Fund (No. 007/2017/A1 and132/2017/A3), Macao SAR, China.

\section{Author contributions}

All authors commented on the manuscript.

\section{Competing interests}

The authors declare no competing financial interests.

\section{Supplementary information}

Supplementary information for this paper is available at https://doi.org/10.29026/oea.2022.200046 